
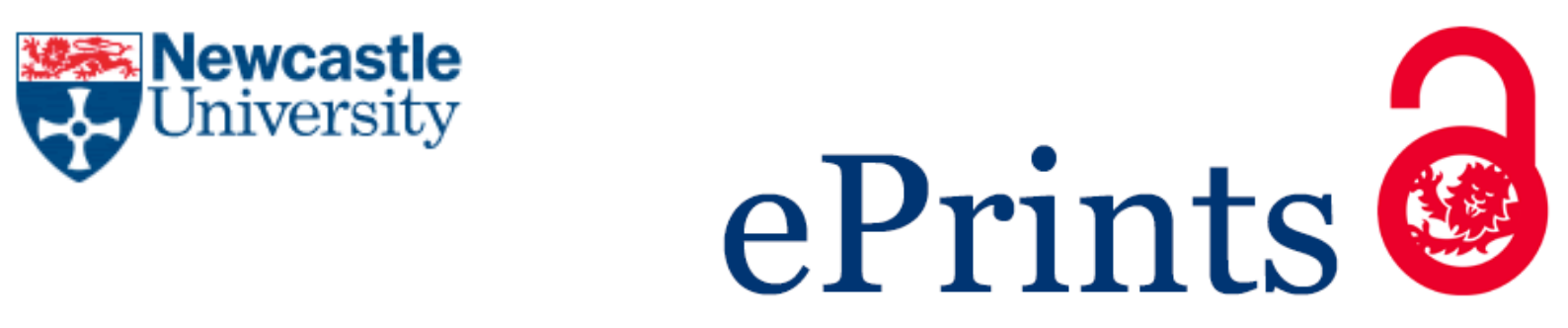

\author{
Mathieu L, Costa AL, Le Bachelier C, Slama A, Lebre AS, Taylor RW, \\ Bastin J, Djouadi F. \\ $\underline{\text { Resveratrol attenuates oxidative stress in mitochondrial Complex I }}$ \\ deficiency: Involvement of SIRT3. \\ Free Radical Biology and Medicine 2016, 96, 190-198.
}

\title{
Copyright:
}

(C) 2016. This manuscript version is made available under the CC-BY-NC-ND 4.0 license

DOI link to article:

http://dx.doi.org/10.1016/j.freeradbiomed.2016.04.027

Date deposited:

$03 / 11 / 2016$

Embargo release date:

25 April 2017

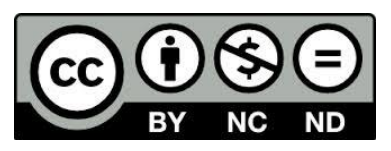

This work is licensed under a

Creative Commons Attribution-NonCommercial-NoDerivatives 4.0 International licence 


\section{Resveratrol attenuates oxidative stress in mitochondrial Complex I deficiency: involvement of SIRT3}

Lise Mathieu ${ }^{1}$, Alexandra Lopes Costa ${ }^{1}$, Carole le Bachelier ${ }^{1}$, Abdelhamid Slama $^{2}$, Anne-Sophie Lebre ${ }^{3}$, Robert W. Taylor ${ }^{4}$, Jean Bastin ${ }^{1}$, Fatima Djouadi ${ }^{1}$.

${ }^{1}$ INSERM UMRS 1124, Université Paris Descartes, 75006 Paris, France;

${ }^{2}$ Laboratoire de Biochimie, AP-HP Hôpital, 94270 Kremlin Bicêtre, France

${ }^{3} \mathrm{CHU}$ Reims, Hôpital Maison Blanche, Pôle de biologie, Service de génétique, 51092 Reims, France

${ }^{4}$ Wellcome Trust Centre for Mitochondrial Research, Institute of Neuroscience, The Medical School, Newcastle University, NE2 4HH Newcastle upon Tyne, UK

Abbreviated title: Resveratrol against ROS in Complex I disorders.

Key terms: Complex I deficiency, oxidative stress, mitochondrial diseases, pharmacological therapy, resveratrol, SOD2, SIRT3

\section{Number of figures and tables: 5}

Corresponding author:

Fatima Djouadi, PhD

INSERM U1124

Université Paris Descartes

45 , rue des Saints-Pères

75270 Paris cedex 06

France

Phone: 33-1-42862219

Fax: 33-1-42863868

e-mail: fatima.djouadi@inserm.fr 
Disclosure statement: the authors have nothing to disclose

\section{Abstract}

The pathophysiological mechanisms underlying Complex I (Cl) deficiencies are understood only partially which severely limits the treatment of this common, devastating, mitochondrial disorder. Recently, we have shown that resveratrol (RSV), a natural polyphenol, has beneficial effects on $\mathrm{Cl}$ deficiency of nuclear origin. Here, we demonstrate that RSV is able to correct the biochemical defect in oxygen consumption in five of thirteen $\mathrm{Cl}$-deficient patient cell lines. Other beneficial effects of RSV include a decrease of total intracellular ROS and the up-regulation of the expression of mitochondrial superoxide dismutase (SOD2) protein, a key antioxidant defense enzyme. The molecular mechanisms leading to the up-regulation of SOD2 protein expression by RSV require the estrogen receptor (ER) and the estrogen-related receptor alpha (ERR $\alpha)$. Although RSV increases the level of SOD2 protein in patients' fibroblasts, the enzyme activity is not increased, in contrast to normal fibroblasts. This led us to hypothesize that SOD2 enzyme activity is regulated post-translationally. This regulation involves SIRT3, a mitochondrial NAD ${ }^{+}$-dependent deacetylase and is critically dependent on $\mathrm{NAD}^{+}$levels. Taken together, our data show that the metabolic effects of RSV combined with its antioxidant capacities makes RSV particularly interesting as a candidate molecule for the therapy of $\mathrm{Cl}$ deficiencies. 


\section{Introduction}

Isolated mitochondrial complex I (Cl) deficiency is one of the most common causes of mitochondrial disease. It originates from mutations in either mitochondrial or nuclear DNA [1]. The defect of Cl usually causes progressive neuro-degenerative disorders, which explains the variety of clinical presentations associating encephalo-myopathy, developmental delay, hypotonia, seizure, cardiomyopathy, optic atrophy and other organ involvement [2]. Interestingly, $\mathrm{Cl}$ dysfunction also has been implicated in lateonset neurodegenerative disorders such as Parkinson's disease [3]. In recent years, significant advances have been made in the diagnosis and characterization of these mitochondrial disorders. In particular, the implementation of new sequencing methods has facilitated the identification of numerous disease genes [4]. In contrast, the physiopathology that explains the cellular dysfunctions remains poorly understood which limits treatment options.

Among the possible pathophysiological mechanisms that could link decreases in activity of $\mathrm{Cl}$ to cellular dysfunction, an imbalance between the production of endogenous reactive oxygen species (ROS) and the antioxidant defense system has been proposed [5]. Although low concentrations of ROS can serve as a second messenger to regulate diverse physiological functions, overproduction of ROS by mitochondria, the main source of intracellular ROS, may lead to oxidative damage. Thus, development of drugs, which can maintain ROS homeostasis, might be useful for alleviating the symptoms associated with $\mathrm{Cl}$ deficiency $[6,7]$.

Resveratrol is a polyphenolic compound that is proposed to have a wide range of beneficial effects on health [8]. Its numerous biological properties have been studied extensively to understand these effects. In animal models and in cell cultures, resveratrol exerts diverse biological effects probably due to the fact that it has many molecular targets [9]. Recently, we have shown that resveratrol corrects the defects in oxygen consumption in fibroblasts from some patients with moderate $\mathrm{Cl}$-deficiency, via the estrogen receptor (ER) and the estrogen-related receptor alpha (ERR $\alpha$ ) pathways [10]. Resveratrol also has been shown to exert beneficial neuroprotective effects through its antioxidant capacity [11]. However, the precise molecular mechanisms, which underlie the antioxidant effects of RSV, are not completely understood. RSV may have direct scavenging properties or it may induce endogenous anti-oxidative enzymes [11]. Among these anti-oxidative enzymes, the mitochondrial manganese superoxide dismutase (SOD2) is the first-line antioxidant defense enzyme that protects cells from oxidative stress generated in the mitochondria. Sirtuin 3 (SIRT3), a member of the class III histone deacetylase family that resides primarily in mitochondria, may regulate many aspects of oxidative metabolism [12]. Interestingly, several recent studies showed that SIRT3 is a major regulator of the mitochondrial adaptive response to stress, in particular, through activation of SOD2 by deacetylation [13-15]. In this study, we tested the hypothesis that RSV can correct $\mathrm{Cl}$ deficiency in skin fibroblasts from patients by 1) relieving the metabolic blockade 
and 2) alleviating oxidative stress. Our results shed new light on the molecular mechanisms underlying these effects. 


\section{Materials and methods}

\section{Cell culture}

Complex I-deficient human fibroblasts were obtained from different reference centers for the investigation and biochemical/molecular diagnosis of mitochondrial disorders. All fibroblasts were obtained from patients with recessively inherited isolated $\mathrm{Cl}$ deficiency, which originated from mutations in nuclear encoded $\mathrm{Cl}$ subunit genes, as summarized in Table I. Unless otherwise mentioned, fibroblasts from patients and normal, control individuals were cultured at $37^{\circ} \mathrm{C}, 5 \% \mathrm{CO}_{2}$ in $\mathrm{RPMI}^{\text {with }}$ Glutamax (Gibco) supplemented with $10 \%$ (V/V) fetal bovine serum and $0.2 \%$ (V/V) primocin (Invivogen). For treatment, the medium was removed and vehicle (0.04\% DMSO), RSV (75 $\mu$ M trans-RSV, Cayman

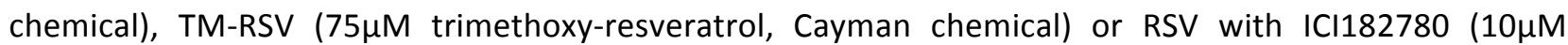

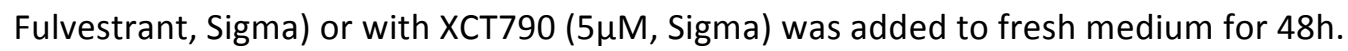

\section{Oxygen consumption}

Maximal Oxygen Uptake Rates (OUR) were measured using Oxoplates ${ }^{\circledR}$ OP96U, 96-well microplates with integrated optical oxygen sensors (PreSens, Germany), as previously described [10].

\section{Measurement of cellular ATP}

Fibroblasts were seeded (4000 cells/well), in triplicate, into 96-well culture plates. The next day, the medium was removed and replaced by glucose-free medium, 10\% (V/V) fetal bovine serum, 0.2\% (V/V) primocin and supplemented with $5 \mathrm{mM}$ galactose. This procedure reveals putative differences in ATP content, since cells grown in galactose rely, mostly, on oxidative phosphorylation to produce their ATP [16, 17]. ATP levels were measured using the Luminescent ATP detection assay kit according to the manufacturer's instructions (Abcam).

\section{Complex I enzyme activity}

Complex I enzyme activity was assayed according to the spectrophotometric method described in [18].

\section{Measurement of cellular reactive oxygen species}

Total levels of cellular ROS were measured using 2',7'-dichlorodihydrofluorescein-diacetate (H2DCFDA, Invitrogen) according to the manufacturer's recommendations. Briefly, experiments were performed in 24-wells in which cells were incubated with $5 \mu \mathrm{M} \mathrm{H} 2 \mathrm{DCFDA}$ for $40 \mathrm{~min}$ at $37^{\circ} \mathrm{C}$. Cells were subsequently washed with PBS, lysed and transferred to a 96-well black plate for measurement of fluorescence intensity with a plate reader (infinite ${ }^{\circledR} \mathrm{M} 200$, Tecan). The results were normalized to the amount of protein in each well. 


\section{SIRT3 enzyme activity}

Mitochondria were isolated using a mitochondria isolation kit for cultured cells (Abcam) according to the manufacturer's instructions. The activity of mitochondrial SIRT3 enzyme was then measured using a fluorometric SIRT3 activity assay kit (Abcam) according to the manufacturer's recommendations.

\section{Western blot analysis}

Western blots were performed as previously described [10]. Briefly, cell protein extracts $(15-20 \mu \mathrm{g})$ were resolved by $12 \%$ SDS-PAGE and transferred to PVDF membranes. Proteins were detected with the following antibodies: SOD2 (Abcam); SIRT3 (Cell Signaling) and $\beta$-actin (Millipore).

\section{Superoxide dismutase 2 enzyme activity}

Determination of superoxide dismutase (SOD) enzyme activity in fibroblasts was performed according to the spectrophotometric method of Paoletti et al, with minor modifications [19].

\section{RNA interference}

Small interfering RNAs (siRNAs) targeting the SIRT3 sequence and control non-target siRNAs were obtained from Dharmacon (ON-TARGET plus SMARTpool). Fibroblasts were transfected with 30nM siRNAs 48h prior to RSV treatment, using Lipofectamine ${ }^{\circledR}$ RNAiMAX Reagent according to the manufacturer's instructions.

\section{Statistical analysis}

The results are presented as the mean \pm SEM. Differences between groups were analyzed by paired or unpaired Student's $t$ test for the comparison of two groups, or by one-way ANOVA and the Fisher test for comparison of three or more groups. $\mathrm{P}<0.05$ was considered significant. 


\section{Results}

Resveratrol corrects $\mathrm{Cl}$ deficiencies and decreases intracellular ROS levels

As shown in Fig. 1A, under basal conditions, the fibroblasts from patients generally exhibited a significant decrease in oxygen consumption, which ranged from extremely severe (P5) to relatively moderate (P10, $25 \%$ as compared to control). This is consistent with their $\mathrm{Cl}$ deficiencies. In control cells, RSV increased OUR 1.5-fold. After exposure to RSV, 8 of the 13 patient cell lines exhibited marked increases in OUR, from $+31 \%$ (Patient 8 ) to $+170 \%$ (Patient 3 ). Notably, nearly normal OUR values were reached in five cell lines (P2, P4, P8, P10 and P13). The relative amounts of ATP were measured in some of the patients' fibroblasts. $\mathrm{Cl}$ deficient cells had a 15 to $25 \%$ reduction of cellular ATP content $(p<0.05)$ as compared to control cells (Fig. 1B). Treatment with RSV increased the level of ATP $23 \%$ in the control cells as well as slightly, but significantly, in the patients' cells. For the rest of the study, we decided to use the Cl-deficient cells in which RSV treatment nearly normalized OUR levels, that is P2, P4, P8, P10 and P13. The residual Cl enzyme activity (in the absence of RSV) in these cell lines ranged from $28 \%$ (P8) to $56 \%$ (P10) of that in the control cells (Fig. 1C). After treatment with RSV, the Cl activity was significantly increased in the control cells (+32\%) as well as in all the Cl-deficient cells (from $+33 \%$ in $\mathrm{P} 2$ and $\mathrm{P} 4$ to $+91 \%$ in $\mathrm{P} 13$ ).

Excessive ROS production by defective mitochondria often has been proposed as a possible mechanism to explain the physiopathology of respiratory chain disorders. This led us to investigate whether abnormal ROS levels existed in the same five cell lines (P2, P4, P8, P10 and P13). We found that all five Cl-deficient cell lines exhibited levels of intracellular ROS that were significantly higher than those found in the control cells, although to different extents (Fig. 1D). P10 displayed a 1.3-fold increase in ROS production whereas a 6-fold increase was found for P8. In all five cell lines, treatment with RSV significantly decreased the levels of ROS and, in four of those ROS levels were not significantly different from control vehicle-treated cells.

Resveratrol increases the amount of SOD2 protein via the ER and ERR $\alpha$ signaling pathways in human fibroblasts

Since manganese superoxide dismutase (SOD2) is known to play a crucial role in controlling the levels of ROS and since it is inducible by RSV in several cell types we sought to determine whether this enzyme was involved in the decrease in the levels of ROS that was observed in the RSV-treated fibroblasts. First, we studied the effects of RSV on the amount of SOD2 protein in the control cells and in the five patient cell lines. As shown in Fig.2A, untreated Cl-deficient cells had amounts of SOD2 that were, as compared to the levels in control cells, comparable (P2), reduced (P4 and P8) or increased (P10 and P13). However, in all the cell lines, treatment with RSV markedly increased the amount of SOD2 (from $+25 \%$ in P13 to $+90 \%$ in $\mathrm{P} 4$ in the $\mathrm{Cl}$-deficient cells and $+89 \%$ in the control cells). 
We next investigated the molecular mechanisms that could account for the increase of SOD2 protein in response to RSV in human fibroblasts. In a previous study, we proposed that RSV (75 $\mu \mathrm{M})$, acting as a phytoestrogen, up-regulated mitochondrial oxygen consumption through the ER [10]. Since SOD2 is a mitochondrial protein, it is plausible that the ER also might be involved in the regulation of SOD2. To test this hypothesis, we treated control fibroblasts with the specific ER antagonist ICI182780. We found that the marked increase in the amount of SOD2 protein that was triggered by RSV was fully abolished by the antagonist (Fig. 2B), which clearly indicates the involvement of ER. However, to our knowledge, the ER does not directly regulate SOD gene expression. This led us to envisage another hypothesis, which involves the ERR $\alpha$. We previously reported that RSV, through its action on the ER, is a potent inducer of the master regulator of mitochondrial respiratory chain and biogenesis, namely the ERR $\alpha$. The increase in the amount of SOD2 protein in the cells produced by RSV was totally prevented by the specific ERR $\alpha$ inverse agonist XCT790 (Fig. 2C). This result demonstrates the participation of the ERR $\alpha$ in the RSV signaling cascade.

\section{Resveratrol-induced SOD2 enzyme activity is under SIRT3 control.}

We next investigated whether the increase of SOD2 protein resulted in an increase in SOD2 enzyme activity, as might be anticipated. We found that the increase of SOD2 protein following treatment with RSV was accompanied, indeed, by a corresponding increase in the SOD2 enzymatic activity in the control cells $(+38 \%)$, and, to a lesser extent, in P13 $(+18 \% ; \mathrm{p}<0,05)($ Fig. $3 \mathrm{~A})$. However, in the other four $\mathrm{Cl}-$ deficient cell lines, there was, surprisingly, no increase in SOD2 enzyme activity following treatment with resveratrol. This puzzling observation could be explained if SOD2 is subjected to a post-translational modification. We hypothesized that if this is the case, the post-translational mechanism, which increases SOD2 enzyme activity in control cells, might be dysfunctional in some $\mathrm{Cl}$-deficient cells. In this respect, several recent articles have shown that Sirtuin-3 (SIRT3), a major mitochondrial NAD dependent protein deacetylase, directly activates SOD2 via protein/lysine deacetylation [13-15]. Of crucial importance is the fact that SIRT3 is a $N A D^{+}$dependent protein. Since the available $N A D^{+}$pool might be a limiting factor in some $\mathrm{Cl}$-deficient cells, this could explain the absence of increase of SOD2 activity in these cells following treatment with RSV. Thus, to determine whether the increase in SOD2 enzyme activity is dependent upon SIRT3 in human fibroblasts, we inactivated SIRT3 in control cells by knock down and measured the SOD2 enzyme activity following treatment with RSV. The suppression of SIRT3 expression by specific siRNAs was confirmed by western blot (Fig. 3B). Knocking down SIRT3 prevented the increase in SOD2 enzyme activity due to treatment with RSV in control cells (Fig. 3C), which indicates that SIRT3, indeed, is involved in the signaling pathway that mediates the effects of RSV. 
RSV significantly upregulated SIRT3 protein levels in control cells (Fig. 3B). Therefore, we hypothesized that the absence of increase in SOD2 enzyme activity in response to treatment with RSV in Cl-deficient cells was due to an absence of increase in SIRT3 protein levels. However, western blot analysis showed that treatment with RSV significantly increased SIRT3 protein levels in control cells and in all the $\mathrm{Cl}$ deficient cells (Fig. 3D). Taken together, the results support the notion that the lack of increase in SOD2 activity in the $\mathrm{Cl}$-deficient cells following treatment with resveratrol could be due to defective SIRT3 activity, possibly linked to the low $\mathrm{NAD}^{+}$availability in these cells. Consistent with this hypothesis, we found no significant increase in SIRT3 enzyme activity in patients' cells following treatment with RSV, whereas control fibroblasts treated with RSV exhibited a $39 \%$ increase in enzyme activity (Fig. 3E). Finally, we investigated the possible involvement of ERR $\alpha$ in the up-regulation of SIRT3 protein following treatment with RSV. The increase in the amount of SIRT3 protein observed in response to treatment of control cells with RSV was fully abolished by the ERR $\alpha$ inverse agonist XCT790 (Fig. 3F). This strongly suggests that ERR $\alpha$ is involved in the signal transduction pathway.

\section{Resveratrol but not Trimethoxy-resveratrol decreases ROS levels}

On the basis of our results, we propose a signaling cascade (Fig. 4A), which accounts for the effects of RSV on SOD2 and SIRT3 in human fibroblasts. However, if this scheme is correct the decrease in the levels of ROS following treatment of Cl-deficient fibroblasts with RSV (Fig. 1C) cannot be explained by the scavenging action of SOD2, which is, potentially, hampered by the low availability of NAD'. Therefore, we investigated whether the action of RSV on the levels of ROS also could be due, in part, to its direct antioxidant properties which are linked to the presence of the phenol rings in the chemical structure. To address this question, we compared the effects of treatments with RSV and TM-RSV on the levels of ROS in control and $\mathrm{Cl}$-deficient cells. TM-RSV is a compound in which the three hydroxyl groups have been replaced by three methoxy groups, which strongly reduces the intrinsic antioxidant properties of this molecule as compared to RSV (Fig. 4B). We found that TM-RSV failed to decrease the levels of ROS in both control and $\mathrm{Cl}$-deficient cells (Fig. 4C), which supports the hypothesis that the phenol ring structure is essential for the protective effects of RSV. 


\section{Discussion}

Patients with isolated $\mathrm{Cl}$ deficiency due to mutations in nuclear genes often develop severe, complex and rapidly fatal phenotypes, with many organs involved, including the brain and central nervous system [2]. The excessive production of ROS, which leads to oxidative damages in affected tissues, has often been proposed to explain the physiopathology of these devastating disorders [7]. Indeed, Verkaart et al. [5] demonstrated that superoxide production was increased in skin fibroblasts from several children with $\mathrm{Cl}$ deficiency and suggested that the extent of increase was inversely proportional to the amount of $\mathrm{Cl}$ residual activity. In this study, we have confirmed that the production of ROS is increased in $\mathrm{Cl}$-deficient cell lines, but not to the same extent in all cases. Nevertheless, in our study, the highest level of ROS was found in the cells of patient (P8), which also exhibited the lowest residual $\mathrm{Cl}$ activity. However, since the level of ROS in the cells is the result of both ROS production and ROS scavenging, the antioxidant defense system, in which SOD2 plays a crucial role, is an important participant in the determination of this fine balance. Thus, in our series of patients' fibroblasts, we found evidence that the extent of increase in ROS is also inversely proportional to the SOD2 enzyme activity, as shown by patients P8 and P10. .

Given the compelling evidence that RC dysfunction leads to oxidative stress, it was legitimate that several laboratories tested diverse antioxidants for the prevention of oxidative damage [6, 7]. Our choice to investigate the effects of resveratrol is justified for several reasons. First, in a previous study, we showed that exposure to RSV corrects $\mathrm{Cl}$ deficiency in the cell lines of some patients [10]. Using a new panel of $\mathrm{Cl}$ deficient cells, our work here extends the notion that RSV can improve and even correct the defect in RC flux in cell lines harboring mutations in nuclear-encoded genes for $\mathrm{Cl}$ subunits. Second, RSV has been shown to be a ROS scavenger via its direct antioxidant effects as a polyphenolic compound [20]. However, in the context of its protective effects against oxidative damages, RSV has been studied mainly for its ability to up-regulate the expression of several free radical scavenging enzymes [21-23]. RSV induces the expression of SOD2 in several rodent organs including the brain [24], and in several cell lines including neuronal cells $[21,22]$. Our results show that RSV stimulates SOD2 protein expression in both control and $\mathrm{Cl}$-deficient fibroblasts in agreement with these results.

We unraveled the molecular mechanisms that might explain the increase of SOD2 at the protein level in human fibroblasts. We showed that the ER was involved by demonstrating that the specific ER antagonist ICI182780 blocked the increase in SOD2 protein induced by treatment with RSV. Our results are consistent with those of Robb and Stuart [22], which suggest that RSV interacts with the ER to upregulate SOD2 in several cell lines. However, to our knowledge, our data is first that has permitted the entire signaling pathway to be deciphered. Indeed, since no ER-responsive element has been identified in the promoter region of SOD2, the effect of the ER on the SOD2 gene must be indirect. In our previous study, we proposed that RSV, after interacting with the ER, up-regulated the expression of the orphan receptor ERR $\alpha$ and that this accounted for the coordinated increase in expression that was observed for all of the 
mitochondrial RC proteins in treated cells [10]. Since SOD2 is a mitochondrial protein and since an ERR $\alpha$ responsive element has been identified in its promoter [25], we tested the hypothesis that ERR $\alpha$ participates in the signaling pathway that leads to the increase in SOD2 protein. The use of the inverse agonist XCT790 confirmed the involvement of the ERR $\alpha$ and led us to propose a scheme, presented Fig. 4A, for the regulation of SOD2 expression.

Surprisingly, the measurements of SOD2 enzyme activity revealed that treatment with RSV did not increase SOD2 activity in most $\mathrm{Cl}$-deficient cells, despite increases in SOD2 protein. In this context, Robb et al. [23] have proposed recently that functional mitochondria are necessary since the induction of SOD2 protein by RSV was accompanied by increases in SOD2 enzyme activity in normal PC3 cells but not in rho ${ }^{0}$ PC3 cells. This, naturally, led us to hypothesize a role for SIRT3 since this NAD ${ }^{+}$-dependent sirtuin recently has been found to participate in a novel post-translational regulation of SOD2. Indeed, studies from several laboratories have suggested that SIRT3 binds to, de-acetylates, and activates SOD2 to scavenge ROS in response to various stresses [13-15]. In these studies, two crucial results are 1) that over expression of SOD2 protein reduces cellular ROS only modestly, and 2) that SIRT3 activity is mandatory for the induction of SOD2 activity by deacetylation. Importantly, the removal of $\mathrm{NAD}^{+}$, or the addition of a sirtuin inhibitor, prevented the deacetylation of SOD2 and its subsequent activation [13-15].

We found here that although $\mathrm{Cl}$ enzyme activities were significantly increased by RSV treatment of $\mathrm{Cl}$ deficient cells, they were not restored to the levels of normal control cells. Therefore, we propose that, in the patients' cell lines, SOD2 enzyme activity is not fully restored because the $\mathrm{Cl}$ enzyme activity remains too low to completely replete the mitochondrial $\mathrm{NAD}^{+}$pool, which is limiting for $\mathrm{NAD}^{+}$-dependent sirtuin activity. It would therefore be very interesting to measure the $\mathrm{NAD}^{+}$concentration in isolated mitochondria from control versus patient fibroblasts, especially because it is well admitted that the mitochondrial $\mathrm{NAD}^{+}$pool is distinct from the cytosolic one [26]. Unfortunately, all our attempts to measure the $\mathrm{NAD}^{+}$concentrations in mitochondria-enriched fractions from the various $\mathrm{Cl}$-deficient patient fibroblasts were hampered by the limited amount of cells obtained, due to their slow growth rates. Nevertheless, it is generally acknowledged that inborn Cl-deficiency leads to impaired NADH oxidation and to decreased $\mathrm{NAD}^{+}$levels in the mitochondrial compartment. In line with this, and in keeping with our hypothesis, we found that SIRT3 enzyme activity was not increased after treatment of $\mathrm{Cl}$-deficient cells with RSV, whereas a significant increase was measured in control cells treated with RSV.

These results are in line with those of recent articles, in which different authors highlight the importance of levels of $\mathrm{NAD}^{+}$in mitochondrial disorders and surmise that mitochondrial dysfunction could be ameliorated by replenishing $\mathrm{NAD}^{+}$levels [27-29]. Given the current growing interest of researchers in sirtuins, various strategies have been tested in attempts to raise $\mathrm{NAD}^{+}$pool at the cell level, or in specific cell compartments, including mitochondria $[26,30]$. These approaches relied either on supplementation with $\mathrm{NAD}^{+}$precursors, such as nicotinamide riboside (NR), nicotinamide mononucleotide (NMN), nicotinic 
acid (NA), or nicotinamide (NAM), or were based on treatment with pharmacological inhibitors of Poly(ADP ribose) polymerases (PARPs) [31]. Overall, variable results were reported depending on the cell or animal model considered. Thus, in $\mathrm{Cl}$-deficient fibroblasts, Pirinen et al. demonstrated marked increases in cellular NAD ${ }^{+}$after treatment by MRL-45696 (a PARP1/2 inhibitor) [32], whereas, Felici et al. reported no changes after treatment by another PARP inhibitor (PJ34) [28]. Altogether, these contradictory results also point out that the functioning and balance of $\mathrm{NAD}^{+}$biosynthetic machinery is complex, and still poorly understood [26, 30, 31,33]. For instance, it is admitted that the mitochondrial $\mathrm{NAD}^{+}$pool is relatively distinct from that of the rest of the cell, and some data suggest that mitochondria might have their own $\mathrm{NAD}^{+}$biosynthetic machinery, but the question of how mitochondria maintain their $\mathrm{NAD}^{+}$pool, either by synthetic pathways or by exchanges with the cytoplasmic compartment, is not fully clarified. Importantly, these notions are likely complicated in the patients' fibroblasts since $\mathrm{Cl}$ deficiency leads to a permanent imbalance in $\mathrm{NAD}^{+} / \mathrm{NADH}$ ratio, which might induce chronic adaptive changes in the $\mathrm{NAD}^{+}$biosynthetic pathways. Thus, although $\mathrm{NAD}^{+}$replenishment might form the basis for novel promising therapies, there is to date no consensus on the strategy to use in order to boost cellular or mitochondrial $\mathrm{NAD}^{+}$levels.

The importance of SIRT3 as a pivotal actor in mitochondrial functions such as ATP production recently has been highlighted in several reviews $[12,34,35]$. As previously mentioned, SIRT3 also has been proposed to modulate the response to oxidative stress by reducing ROS production and, thus, plays a key role in the regulation of mitochondrial ROS homeostasis. In this context, the finding that resveratrol induces the expression of SIRT3 is important. However, our results also demonstrate that the up-regulation of SIRT3 expression is not sufficient if the production of $\mathrm{NAD}^{+}$is compromised. In this study, we also have unraveled the molecular mechanisms, which underlie the increase in the level of SIRT3 protein. We have demonstrated the participation of the orphan receptor ERR $\alpha$ in the signaling cascade targeted by RSV, in agreement with the demonstration of an ERR $\alpha$ binding site in the promoter region of the SIRT3 gene [36]. Although SIRT3-dependent SOD2 activation seems to be inoperative in Cl-deficient cells, RSV nevertheless decreases the levels of ROS in these cells. Among the earliest biological activities attributed to RSV were antioxidant properties that are linked to the phenol ring present in its chemical structure. The substitution in RSV of the hydroxy groups by methoxy groups produces a compound, which has lost it antioxidant properties, and demonstrates that the structure of RSV participates in its protective effects against ROS. Finally, RSV has been reported to have a neuro-protective effect in diverse neurodegenerative disorders such as Parkinson's disease, Alzheimer's disease and Huntington's disease [11, 37], all of which are characterized by mitochondrial defects. The fact that RSV has dual antioxidant properties in addition to being able to stimulate residual RC capacity makes this compound a very nice candidate for the biochemical correction of moderate $\mathrm{Cl}$ deficiency. 


\section{Acknowledgements}

This work was supported by grants of the Association Française contre les Myopathies (AFM) and the Agence Nationale de la Recherche (ANR, grant ANR-09-GENO-024-01). RWT is supported by a Wellcome Trust Strategic Award (096919/Z/11/Z), the MRC Centre for Neuromuscular Diseases (G0601943), the Lily Foundation and the UK NHS Highly Specialized "Rare Mitochondrial Disorders of Adults and Children" Service. 


\section{References}

[1] Tucker, E. J.; Compton, A. G.; Calvo, S. E.; Thorburn, D. R. The molecular basis of human complex I deficiency. IUBMB life 63:669-677; 2011.

[2] Distelmaier, F.; Koopman, W. J.; van den Heuvel, L. P.; Rodenburg, R. J.; Mayatepek, E.; Willems, P. H.; Smeitink, J. A. Mitochondrial complex I deficiency: from organelle dysfunction to clinical disease. Brain : a journal of neurology 132:833-842; 2009.

[3] Papa, S.; De Rasmo, D. Complex I deficiencies in neurological disorders. Trends in molecular medicine 19:61-69; 2013.

[4] Haack, T. B.; Haberberger, B.; Frisch, E. M.; Wieland, T.; luso, A.; Gorza, M.; Strecker, V.; Graf, E.; Mayr, J. A.; Herberg, U.; Hennermann, J. B.; Klopstock, T.; Kuhn, K. A.; Ahting, U.; Sperl, W.; Wilichowski, E.; Hoffmann, G. F.; Tesarova, M.; Hansikova, H.; Zeman, J.; Plecko, B.; Zeviani, M.; Wittig, I.; Strom, T. M.; Schuelke, M.; Freisinger, P.; Meitinger, T.; Prokisch, H. Molecular diagnosis in mitochondrial complex I deficiency using exome sequencing. Journal of medical genetics 49:277-283; 2012.

[5] Verkaart, S.; Koopman, W. J.; van Emst-de Vries, S. E.; Nijtmans, L. G.; van den Heuvel, L. W.; Smeitink, J. A.; Willems, P. H. Superoxide production is inversely related to complex I activity in inherited complex I deficiency. Biochimica et biophysica acta 1772:373-381; 2007.

[6] Roestenberg, P.; Manjeri, G. R.; Valsecchi, F.; Smeitink, J. A.; Willems, P. H.; Koopman, W. J. Pharmacological targeting of mitochondrial complex I deficiency: the cellular level and beyond. Mitochondrion 12:57-65; 2012.

[7] Valsecchi, F.; Koopman, W. J.; Manjeri, G. R.; Rodenburg, R. J.; Smeitink, J. A.; Willems, P. H. Complex I disorders: causes, mechanisms, and development of treatment strategies at the cellular level. Developmental disabilities research reviews 16:175-182; 2010.

[8] Tome-Carneiro, J.; Larrosa, M.; Gonzalez-Sarrias, A.; Tomas-Barberan, F. A.; Garcia-Conesa, M. T.; Espin, J. C. Resveratrol and clinical trials: the crossroad from in vitro studies to human evidence. Current pharmaceutical design 19:6064-6093; 2013.

[9] Kulkarni, S. S.; Canto, C. The molecular targets of resveratrol. Biochimica et biophysica acta 1852:1114-1123; 2015.

[10] Lopes Costa, A.; Le Bachelier, C.; Mathieu, L.; Rotig, A.; Boneh, A.; De Lonlay, P.; Tarnopolsky, M. A.; Thorburn, D. R.; Bastin, J.; Djouadi, F. Beneficial effects of resveratrol on respiratory chain defects in patients' fibroblasts involve estrogen receptor and estrogen-related receptor alpha signaling. Human molecular genetics 23:2106-2119; 2014.

[11] Bastianetto, S.; Menard, C.; Quirion, R. Neuroprotective action of resveratrol. Biochimica et biophysica acta 1852:1195-1201; 2015. 
[12] Giralt, A.; Villarroya, F. SIRT3, a pivotal actor in mitochondrial functions: metabolism, cell death and aging. The Biochemical journal 444:1-10; 2012.

[13] Chen, Y.; Zhang, J.; Lin, Y.; Lei, Q.; Guan, K. L.; Zhao, S.; Xiong, Y. Tumour suppressor SIRT3 deacetylates and activates manganese superoxide dismutase to scavenge ROS. EMBO reports 12:534$541 ; 2011$.

[14] Qiu, X.; Brown, K.; Hirschey, M. D.; Verdin, E.; Chen, D. Calorie restriction reduces oxidative stress by SIRT3-mediated SOD2 activation. Cell metabolism 12:662-667; 2010.

[15] Tao, R.; Coleman, M. C.; Pennington, J. D.; Ozden, O.; Park, S. H.; Jiang, H.; Kim, H. S.; Flynn, C. R.; Hill, S.; Hayes McDonald, W.; Olivier, A. K.; Spitz, D. R.; Gius, D. Sirt3-mediated deacetylation of evolutionarily conserved lysine 122 regulates MnSOD activity in response to stress. Molecular cell 40:893-904; 2010.

[16] Aguer, C.; Gambarotta, D.; Mailloux, R. J.; Moffat, C.; Dent, R.; McPherson, R.; Harper, M. E. Galactose enhances oxidative metabolism and reveals mitochondrial dysfunction in human primary muscle cells. PloS one 6:e28536; 2011.

[17] Golubitzky, A.; Dan, P.; Weissman, S.; Link, G.; Wikstrom, J. D.; Saada, A. Screening for active small molecules in mitochondrial complex I deficient patient's fibroblasts, reveals AICAR as the most beneficial compound. PloS one 6:e26883; 2011.

[18] Janssen, A. J.; Trijbels, F. J.; Sengers, R. C.; Smeitink, J. A.; van den Heuvel, L. P.; Wintjes, L. T.; Stoltenborg-Hogenkamp, B. J.; Rodenburg, R. J. Spectrophotometric assay for complex I of the respiratory chain in tissue samples and cultured fibroblasts. Clinical chemistry 53:729-734; 2007.

[19] Paoletti, F.; Mocali, A. Determination of superoxide dismutase activity by purely chemical system based on NAD(P)H oxidation. Methods in enzymology 186:209-220; 1990.

[20] Caruso, F.; Tanski, J.; Villegas-Estrada, A.; Rossi, M. Structural basis for antioxidant activity of trans-resveratrol: ab initio calculations and crystal and molecular structure. Journal of agricultural and food chemistry 52:7279-7285; 2004.

[21] Fukui, M.; Choi, H. J.; Zhu, B. T. Mechanism for the protective effect of resveratrol against oxidative stress-induced neuronal death. Free radical biology \& medicine 49:800-813; 2010.

[22] Robb, E. L.; Stuart, J. A. Resveratrol interacts with estrogen receptor-beta to inhibit cell replicative growth and enhance stress resistance by upregulating mitochondrial superoxide dismutase. Free radical biology \& medicine 50:821-831; 2011.

[23] Robb, E. L.; Stuart, J. A. The stilbenes resveratrol, pterostilbene and piceid affect growth and stress resistance in mammalian cells via a mechanism requiring estrogen receptor beta and the induction of Mn-superoxide dismutase. Phytochemistry 98:164-173; 2014. 
[24] Robb, E. L.; Winkelmolen, L.; Visanji, N.; Brotchie, J.; Stuart, J. A. Dietary resveratrol administration increases MnSOD expression and activity in mouse brain. Biochemical and biophysical research communications 372:254-259; 2008.

[25] Deblois, G.; Hall, J. A.; Perry, M. C.; Laganiere, J.; Ghahremani, M.; Park, M.; Hallett, M.; Giguere, V. Genome-wide identification of direct target genes implicates estrogen-related receptor alpha as a determinant of breast cancer heterogeneity. Cancer research 69:6149-6157; 2009.

[26] Stein, L. R.; Imai, S. The dynamic regulation of NAD metabolism in mitochondria. Trends in endocrinology and metabolism: TEM 23:420-428; 2012.

[27] Cerutti, R.; Pirinen, E.; Lamperti, C.; Marchet, S.; Sauve, A. A.; Li, W.; Leoni, V.; Schon, E. A.; Dantzer, F.; Auwerx, J.; Viscomi, C.; Zeviani, M. NAD(+)-dependent activation of Sirt1 corrects the phenotype in a mouse model of mitochondrial disease. Cell metabolism 19:1042-1049; 2014.

[28] Felici, R.; Lapucci, A.; Cavone, L.; Pratesi, S.; Berlinguer-Palmini, R.; Chiarugi, A. Pharmacological NAD-Boosting Strategies Improve Mitochondrial Homeostasis in Human Complex IMutant Fibroblasts. Molecular pharmacology 87:965-971; 2015.

[29] Khan, N. A.; Auranen, M.; Paetau, I.; Pirinen, E.; Euro, L.; Forsstrom, S.; Pasila, L.; Velagapudi, V.; Carroll, C. J.; Auwerx, J.; Suomalainen, A. Effective treatment of mitochondrial myopathy by nicotinamide riboside, a vitamin B3. EMBO molecular medicine 6:721-731; 2014.

[30] Canto, C.; Menzies, K. J.; Auwerx, J. NAD(+) Metabolism and the Control of Energy Homeostasis: A Balancing Act between Mitochondria and the Nucleus. Cell metabolism 22:31-53; 2015.

[31] Mouchiroud, L.; Houtkooper, R. H.; Auwerx, J. NAD(+) metabolism: a therapeutic target for age-related metabolic disease. Critical reviews in biochemistry and molecular biology 48:397-408; 2013.

[32] Pirinen, E.; Canto, C.; Jo, Y. S.; Morato, L.; Zhang, H.; Menzies, K. J.; Williams, E. G.; Mouchiroud, L.; Moullan, N.; Hagberg, C.; Li, W.; Timmers, S.; Imhof, R.; Verbeek, J.; Pujol, A.; van Loon, B.; Viscomi, C.; Zeviani, M.; Schrauwen, P.; Sauve, A. A.; Schoonjans, K.; Auwerx, J. Pharmacological Inhibition of poly(ADP-ribose) polymerases improves fitness and mitochondrial function in skeletal muscle. Cell metabolism 19:1034-1041; 2014.

[33] Houtkooper, R. H.; Canto, C.; Wanders, R. J.; Auwerx, J. The secret life of NAD+: an old metabolite controlling new metabolic signaling pathways. Endocrine reviews 31:194-223; 2010.

[34] Chen, Y.; Fu, L. L.; Wen, X.; Wang, X. Y.; Liu, J.; Cheng, Y.; Huang, J. Sirtuin-3 (SIRT3), a therapeutic target with oncogenic and tumor-suppressive function in cancer. Cell death \& disease 5:e1047; 2014. 
[35] $\mathrm{Wu}, \mathrm{Y} . \mathrm{T}$.; $\mathrm{Wu}, \mathrm{S}$. B.; Wei, Y. H. Roles of sirtuins in the regulation of antioxidant defense and bioenergetic function of mitochondria under oxidative stress. Free radical research 48:1070-1084; 2014.

[36] Kong, X.; Wang, R.; Xue, Y.; Liu, X.; Zhang, H.; Chen, Y.; Fang, F.; Chang, Y. Sirtuin 3, a new target of PGC-1alpha, plays an important role in the suppression of ROS and mitochondrial biogenesis. PloS one 5:e11707; 2010.

[37] Chaturvedi, R. K.; Beal, M. F. Mitochondria targeted therapeutic approaches in Parkinson's and Huntington's diseases. Molecular and cellular neurosciences 55:101-114; 2013.

[38] Breningstall, G. N.; Shoffner, J.; Patterson, R. J. Siblings with leukoencephalopathy. Seminars in pediatric neurology 15:212-215; 2008.

[39] Pagniez-Mammeri, H.; Lombes, A.; Brivet, M.; Ogier-de Baulny, H.; Landrieu, P.; Legrand, A.; Slama, A. Rapid screening for nuclear genes mutations in isolated respiratory chain complex I defects. Molecular genetics and metabolism 96:196-200; 2009.

[40] Tuppen, H. A.; Hogan, V. E.; He, L.; Blakely, E. L.; Worgan, L.; Al-Dosary, M.; Saretzki, G.; Alston, C. L.; Morris, A. A.; Clarke, M.; Jones, S.; Devlin, A. M.; Mansour, S.; Chrzanowska-Lightowlers, Z. M.; Thorburn, D. R.; McFarland, R.; Taylor, R. W. The p.M292T NDUFS2 mutation causes complex Ideficient Leigh syndrome in multiple families. Brain : a journal of neurology 133:2952-2963; 2010.

[41] Assouline, Z.; Jambou, M.; Rio, M.; Bole-Feysot, C.; de Lonlay, P.; Barnerias, C.; Desguerre, I.; Bonnemains, C.; Guillermet, C.; Steffann, J.; Munnich, A.; Bonnefont, J. P.; Rotig, A.; Lebre, A. S. A constant and similar assembly defect of mitochondrial respiratory chain complex I allows rapid identification of NDUFS4 mutations in patients with Leigh syndrome. Biochimica et biophysica acta 1822:1062-1069; 2012.

[42] Lebon, S.; Minai, L.; Chretien, D.; Corcos, J.; Serre, V.; Kadhom, N.; Steffann, J.; Pauchard, J. Y.; Munnich, A.; Bonnefont, J. P.; Rotig, A. A novel mutation of the NDUFS7 gene leads to activation of a cryptic exon and impaired assembly of mitochondrial complex I in a patient with Leigh syndrome. Molecular genetics and metabolism 92:104-108; 2007.

[43] Lebon, S.; Rodriguez, D.; Bridoux, D.; Zerrad, A.; Rotig, A.; Munnich, A.; Legrand, A.; Slama, A. A novel mutation in the human complex I NDUFS7 subunit associated with Leigh syndrome. Molecular genetics and metabolism 90:379-382; 2007. 
Table I: Genotypes of the respiratory $\mathrm{Cl}$-deficient patients

\begin{tabular}{|c|c|c|c|c|}
\hline Patients & $\begin{array}{l}\text { Mutated } \\
\text { gene }\end{array}$ & Nucleotide changes & $\begin{array}{c}\text { Amino acid } \\
\text { substitutions }\end{array}$ & References \\
\hline P1 & NDUFV1 & $\begin{array}{l}\text { c. } 1142 A>G, \\
c .1157 G>A\end{array}$ & $\begin{array}{l}\text { p.Gln381Arg, } \\
\text { p.Arg386His }\end{array}$ & this study \\
\hline P2 & NDUFV1 & $\begin{array}{l}\text { c. } 1156 \mathrm{C}>\mathrm{T}, \\
\text { c. } 1156 \mathrm{C}>\mathrm{T}\end{array}$ & $\begin{array}{l}\text { p.Arg386Cys, } \\
\text { p.Arg386Cys }\end{array}$ & [38] \\
\hline P3 & NDUFV2 & $\begin{array}{l}\text { c. } 547 G>A, \\
\text { c. } 207 \text { dup }\end{array}$ & $\begin{array}{l}\text { p.Ala183Thr, } \\
\text { p.Tyr70fs*6 }\end{array}$ & this study \\
\hline P4 & NDUFV2 & $\begin{array}{l}.120+5 \_120+8 \text { delGTAA, } \\
.120+5 \_120+8 \text { delGTAA }\end{array}$ & $\begin{array}{l}\text { p.Gly19_Val40del, } \\
\text { p.Gly19_Val40del }\end{array}$ & this study \\
\hline P5 & NDUFS1 & $\begin{array}{l}\text { c. } 1139 A>T, \\
\text { c. } 63+6 T>G\end{array}$ & p.Asp380Val, & this study \\
\hline P6 & NDUFS1 & $\begin{array}{l}\text { c. } 683 \mathrm{~T}>\mathrm{C}, \\
\text { c. } 755 \mathrm{~A}>\mathrm{G}\end{array}$ & $\begin{array}{l}\text { p.Val228Ala, } \\
\text { p.Asp252Gly }\end{array}$ & [39] \\
\hline P7 & NDUFS2 & $\begin{array}{l}\text { c. } 1237 \mathrm{~T}>\mathrm{C}, \\
\text { c. } 1237 \mathrm{~T}>\mathrm{C}\end{array}$ & $\begin{array}{l}\text { p.Ser413Pro, } \\
\text { p.Ser413Pro }\end{array}$ & this study \\
\hline P8 & NDUFS2 & $\begin{array}{l}\text { c. } 875 \mathrm{~T}>\mathrm{C}, \\
\text { c. } 1328 \mathrm{~T}>\mathrm{A}\end{array}$ & $\begin{array}{l}\text { p.Met292Thr, } \\
\text { p.Met443Lys }\end{array}$ & [40] \\
\hline P9 & NDUFS2 & $\begin{array}{l}\text { c. } 875 \mathrm{~T}>\mathrm{C}, \\
\text { c. } 353 \mathrm{G}>\mathrm{A}\end{array}$ & $\begin{array}{l}\text { p.Met292Thr, } \\
\text { p.Arg118Gln }\end{array}$ & [40] \\
\hline P10 & NDUFS4 & $\begin{array}{l}\text { c.291delG, } \\
\text { c.291delG }\end{array}$ & $\begin{array}{l}\text { p.Trp97X, } \\
\text { p.Trp97X }\end{array}$ & [41] \\
\hline P11 & NDUFS6 & $\begin{array}{l}\text { c. } 67 \mathrm{del} \text {, } \\
\text { c. } 67 \mathrm{del}\end{array}$ & $\begin{array}{l}\text { p.Leu23Trpfs*35, } \\
\text { p.Leu23Trpfs*35 }\end{array}$ & this study \\
\hline P12 & NDUFS7 & $\begin{array}{l}\text { c. } 17-1167 C>G, \\
\text { c. } 17-1167 C>G\end{array}$ & $\begin{array}{l}\text { Ala6_Arg213del, } \\
\text { Ala6_Arg213del }\end{array}$ & [42] \\
\hline P13 & NDUFS7 & $\begin{array}{l}\text { c. } 434 \mathrm{G}>\mathrm{A} \text {, } \\
\text { c. } 434 \mathrm{G}>\mathrm{A}\end{array}$ & $\begin{array}{l}\text { p.Arg145His, } \\
\text { p.Arg145His }\end{array}$ & [43] \\
\hline
\end{tabular}




\section{Figure legends}

Figure 1 Resveratrol can improve $\mathrm{Cl}$ deficiency and ROS accumulation. Experiments were performed in control and $\mathrm{Cl}$-deficient fibroblasts grown for $48 \mathrm{~h}$ in the presence of $75 \mu \mathrm{M}$ resveratrol (black bars), or vehicle (DMSO, white bars). Oxygen Uptake Rates (OUR) per $10^{6}$ cells (A). Amount of cellular ATP (B). Complex I enzyme activity (C). Total cellular reactive oxygen species (D). Control values are from three healthy individuals. The results are means \pm SEM of at least two different experiments. In each experiment, samples were run in triplicate. ${ }^{*} \mathrm{P}<0.05$; ${ }^{* *} \mathrm{P}<0.01 ;{ }^{* * *} \mathrm{P}<0.001$ as compared to vehicletreated fibroblasts. $\S \mathrm{P}<0.05 ; \S \S \mathrm{P}<0.01 ; \S \S \S \mathrm{P}<0.001$ as compared to vehicle-treated control fibroblasts.

Figure 2 The ER and ERR $\alpha$ are involved in the increase of SOD2 protein level triggered by resveratrol. Control and $\mathrm{Cl}$-deficient fibroblasts $(\mathbf{A})$ or control fibroblasts $(\mathbf{B}, \mathbf{C})$ were treated with various compounds

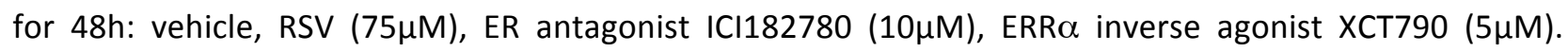
Representative Western-blots and histograms quantifying the amount of SOD proteins are shown. The results are the means \pm SEM for $n=2$ to $n=6(A, B)$ or $n=4$ (C) experiments. In each experiment, samples were run in triplicate. ${ }^{*} \mathrm{P}<0.05 ;{ }^{* *} \mathrm{P}<0.01 ;{ }^{* *} \mathrm{P}<0.001$ versus the respective vehicle-treated fibroblasts. $\S$ $\mathrm{P}<0.05 ; \S \S \mathrm{P}<0.01 ; \S \S \S \mathrm{P}<0.001$ versus control fibroblasts treated with vehicle.

Figure 3 Resveratrol does not increase SOD2 enzyme activity in Cl-deficient fibroblasts: involvement of SIRT3. Control and Cl-deficient fibroblasts (A, D, E) or control cells (B, C, F) were treated $48 \mathrm{~h}$ with various compounds: vehicle, RSV $(75 \mu \mathrm{M})$, XCT790 $(5 \mu \mathrm{M})$. SOD2 enzyme activity in control and $\mathrm{Cl}$-deficient fibroblasts (A). Validation of the SIRT3 knock down (B). SOD2 enzyme activity in control fibroblasts transfected with siNon-Target (NT) or siSIRT3 ( $n=3$ independent experiments) 48 h prior treatment with RSV (C). Histograms of the amount of SIRT3 protein and representative western-blots (D, F). SIRT3 enzyme activity in control and $\mathrm{Cl}$-deficient fibroblasts $(\mathrm{E})$. The results are the means $\pm \mathrm{SEM}$ of at least three experiments. ${ }^{*} \mathrm{P}<0.05 ;{ }^{* * *} \mathrm{P}<0.001$ versus the respective vehicle-treated fibroblasts. $\S \mathrm{P}<0.05 ; \S \S$ $\mathrm{P}<0.01 ; \S \S \S \mathrm{P}<0.001$ versus control fibroblasts treated with vehicle.

Figure 4 Resveratrol chemical structure is important for its antioxidant action in Cl-deficient cells. Proposed mechanisms for the effects of RSV on ROS homeostasis (A). Chemical structures of RSV and trimethoxy-resveratrol (TM-RSV) (B). Control and Cl-deficient fibroblasts were treated with RSV or TMRSV prior the measurement of total levels of cellular ROS using H2DCFDA (C). The results are the means \pm SEM of at least two experiments. In each experiment, samples were run in triplicate. 
${ }^{*} \mathrm{P}<0.05 ;{ }^{* *} \mathrm{P}<0.01$ versus the respective vehicle-treated fibroblasts. $\S \mathrm{P}<0.05 ; \S \S \mathrm{P}<0.01 ; \S \S \S \mathrm{P}<0.001$ versus control fibroblasts treated with vehicle. 


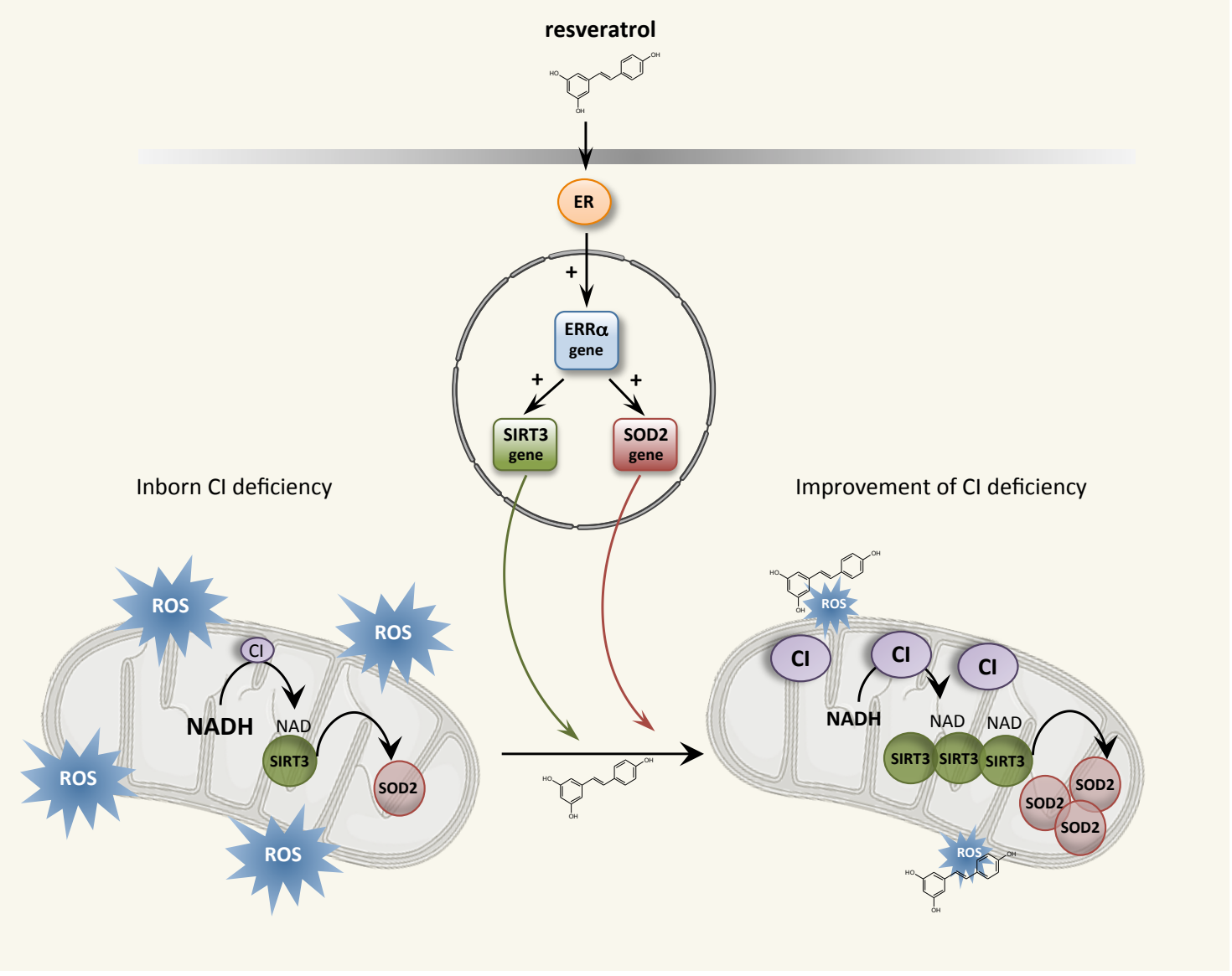

Graphical abstract 
A

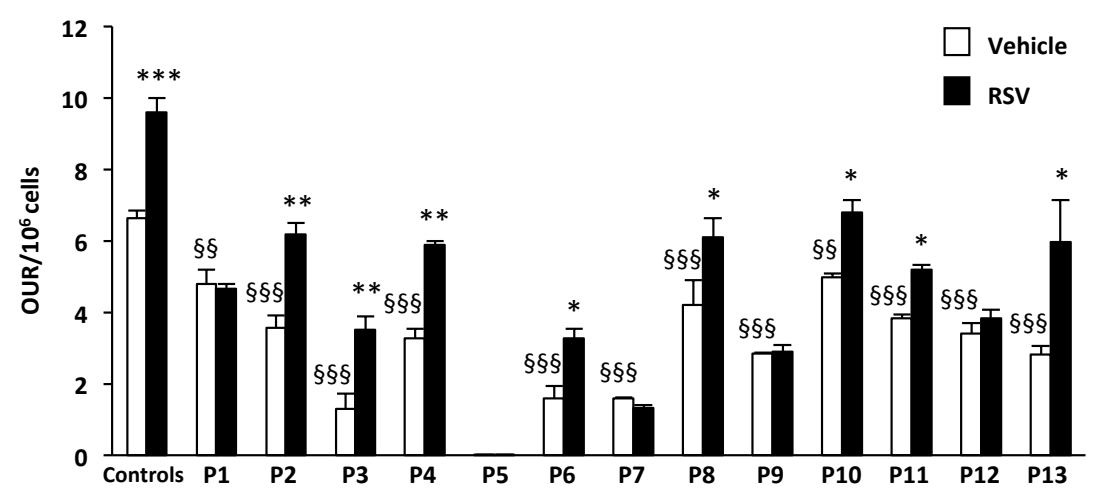

B

C
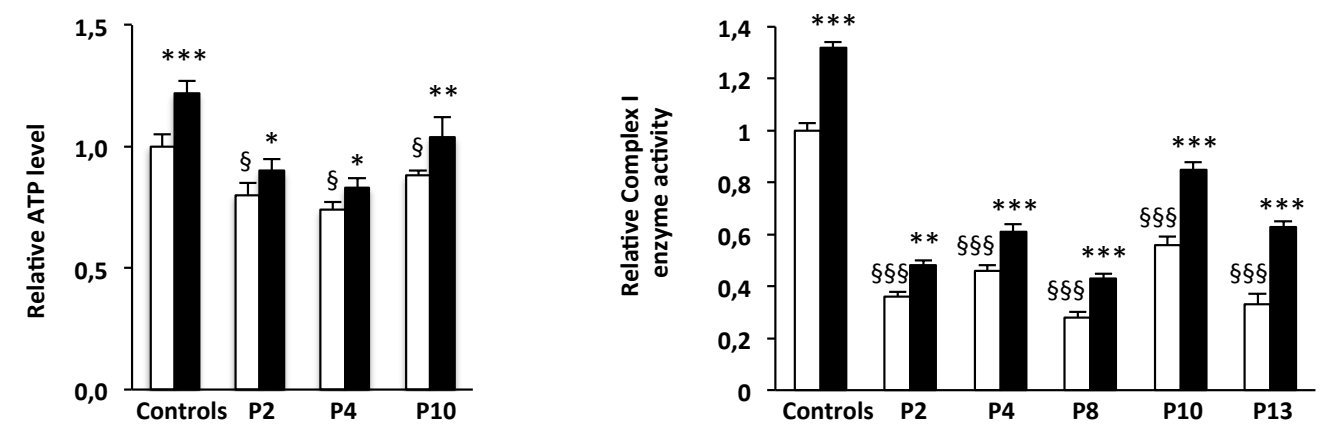

D

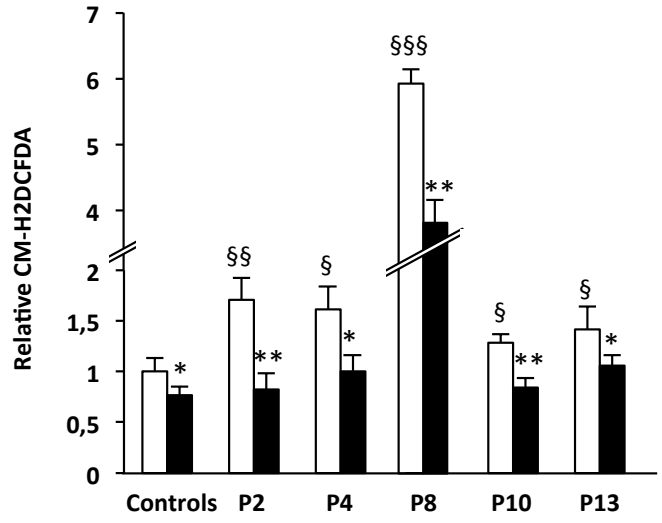

Figure 1 
A
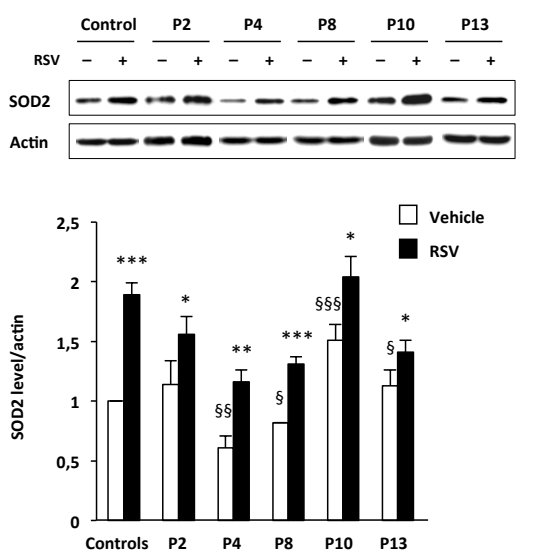

A

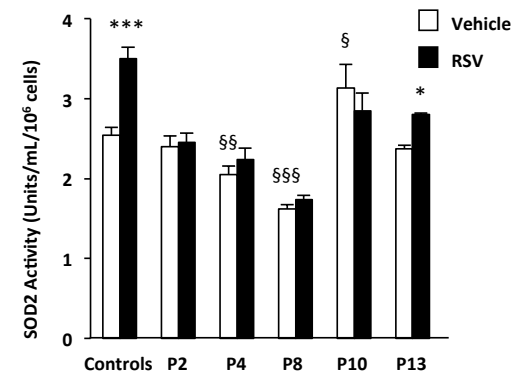

D

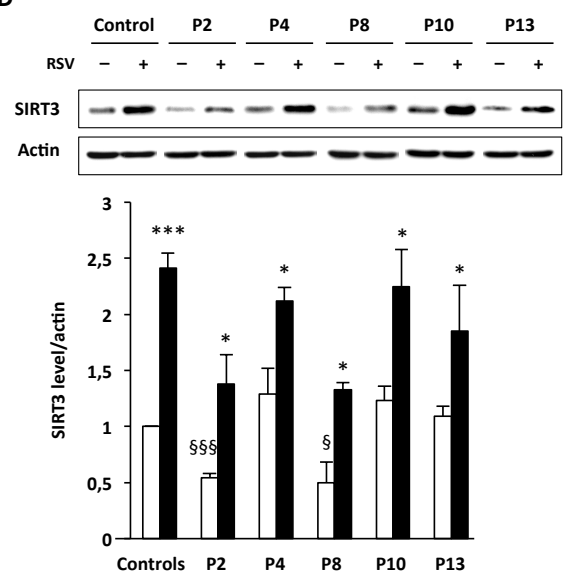

B
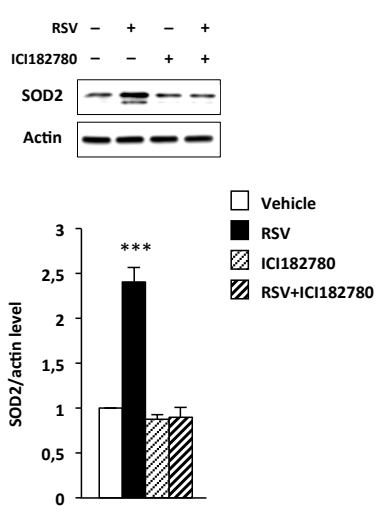

Figure 2 c
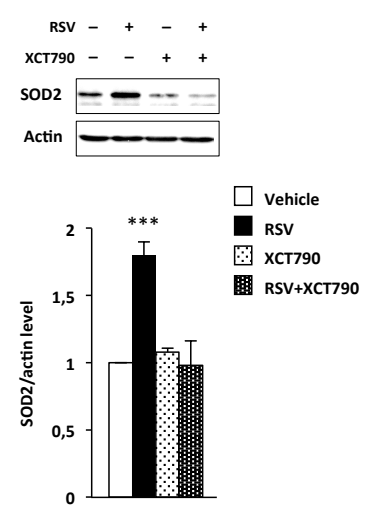

C

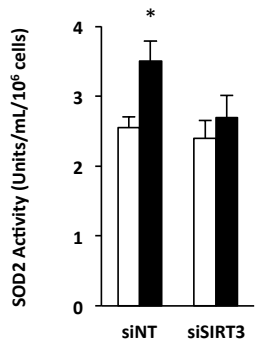

F
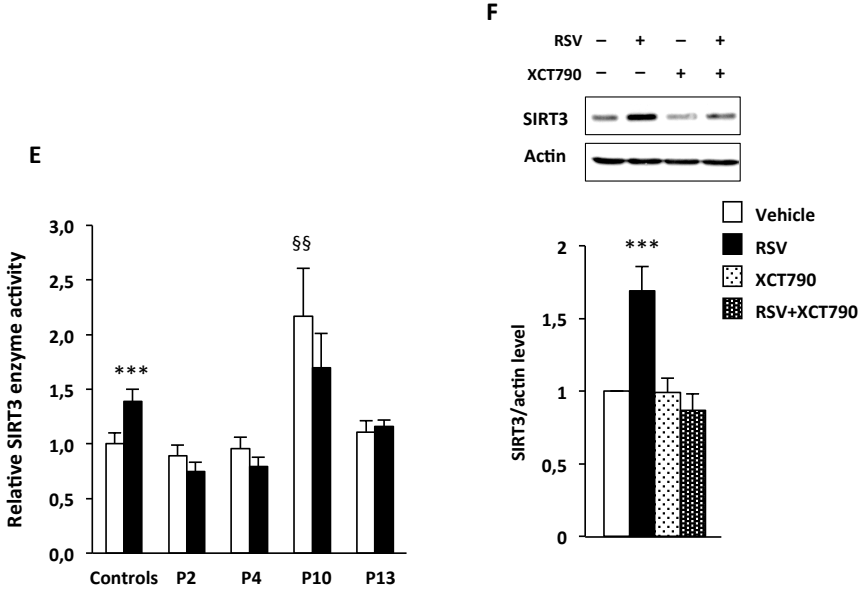

Figure 3 


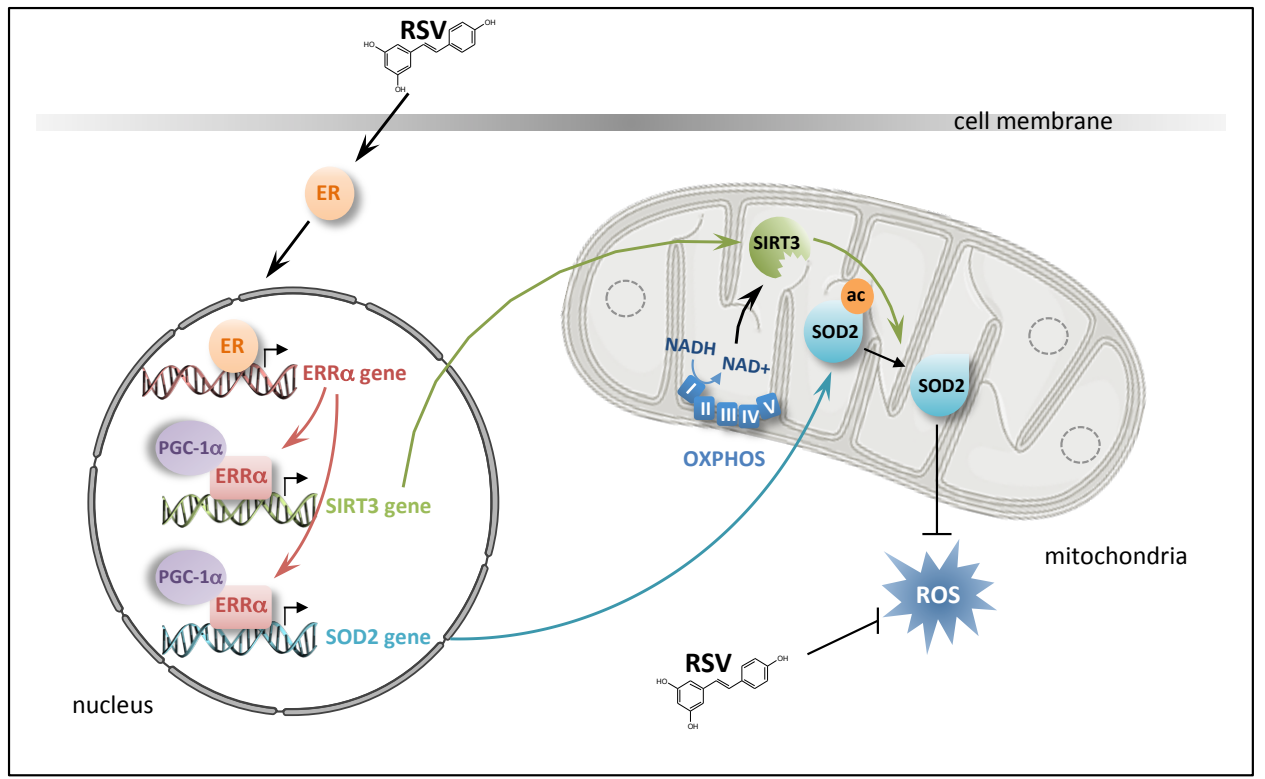

B<smiles>Oc1ccc(/C=C/c2cc(O)cc(O)c2)cc1</smiles>

Resveratrol (RSV)

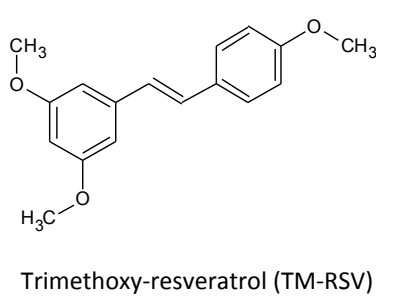

\section{C}

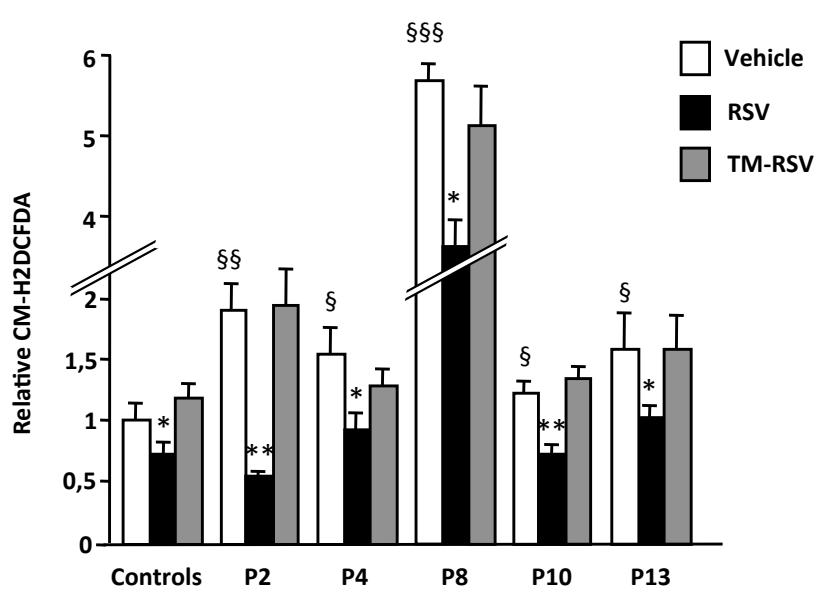

Figure 4 